\title{
Distribution and stability of antisense phosphorothioate oligonucleotides in rodent brain following direct intraparenchymal controlled-rate infusion
}

\author{
William C. Broaddus, M.D., Ph.D., Sujit S. Prabhu, M.D., F.R.C.S.(E), George T. Gillies, Ph.D., \\ Jeffrey Neal, B.S., William S. Conrad, B.S., Zhi-Jian Chen, M.D., Helen Fillmore, Ph.D., and \\ Harold F. Young, M.D.
}

Division of Neurosurgery, Medical College of Virginia, Richmond, VA; and Department of Biomedical Engineering, University of Virginia, Charlottesville, VA

High-flow microinfusion is a novel technique for delivery of compounds directly into the brain parenchyma, bypassing the blood-brain barrier. The feasibility of this technique has been demonstrated with low-molecular-weight compounds, macromolecular dyes, and proteins. Delivery of antisense oligonucleotides into the brain parenchyma represents an additional potential application of this technique not previously described. In this report, the authors examined the distribution and disposition of phosphorothioate oligodeoxynucleotide (PS-ODN) infused for this reason. An 18-mer ${ }^{35}$ S-PS-ODN (molecular weight approximately 6000) was infused over 1 hour into the caudate putamen of Fischer 344 rats. At 1, 6, 12, 24, and 48 hours after beginning the infusion, the brains were extracted and analyzed using quantitative autoradiographic techniques. Cerebrospinal fluid (CSF) was also aspirated from the cisterna magna and analyzed for radioactivity and stability of the ${ }^{35} \mathrm{~S}-\mathrm{PS}-\mathrm{ODN}$. At 1 hour, the infused ODN was uniformly distributed in brain tissue, with a maximum average concentration of $4806.5 \pm$ $210.5 \mathrm{nCi} / \mathrm{g}$. This represents a tissue concentration of $19.2 \pm 0.84 \mu \mathrm{M}$. Extensive spread into surrounding parenchyma was observed over the ensuing 47 hours. The ${ }^{35}$ S-PS-ODN radioactivity peaked in the CSF at the end of the 1-hour infusion, containing $10 \%(50 \pm 20 \mathrm{nCi})$ of the infused radioactivity. Activity then decayed exponentially over 11 hours, stabilizing at a lower CSF content of $0.2 \%(1 \pm 0.1 \mathrm{nCi})$. The volume of distribution $\left(\mathrm{V}_{\mathrm{d}}\right.$ ) was $105 \pm 7.9 \mathrm{~mm}^{3}$ at 1 hour, representing a ratio of $\mathrm{V}_{\mathrm{d}} / \mathrm{V}_{\mathrm{i}}$ (volume of infusion) of 5.2. The $\mathrm{V}_{\mathrm{d}}$ increased to $443.4 \pm 62.3 \mathrm{~mm}^{3}$ at the end of 48 hours, whereas the average minimum tissue concentration decreased from 15.2 to $3.2 \mu \mathrm{M}$. Undegraded 18-mer was seen throughout the 48-hour period using $20 \%$ polyacrylamide/7M urea gel electrophoresis. The animals tolerated the infusion without evidence of toxicity, and minimal structural changes in tissue were observed on histological examination. Thus, PS-ODN can be safely delivered in high concentrations to wide areas of the rat brain by using high-flow microinfusion, and the concentrations remain stable even after 48 hours in situ.

Key Words * antisense oligonucleotide * high-flow microinfusion * autoradiography $*$ gel electrophoresis 
The feasibility of applying high-flow microinfusion techniques for the delivery of low- and high-molecular-weight compounds directly into brain parenchyma has been realized only very recently. $[9,18]$ This technique involves the use of convective flow along pressure gradients established by the infusion process to achieve a wide distribution of solute molecules. This process is distinct from other processes of diffusion in which molecules travel along concentration gradients. The latter processes are more highly sensitive to molecular weight and characterized by rates of migration of solute molecule orders of magnitude slower than convection-enhanced flow.[17,23] The high-flow microinfusion approach is particularly significant because of the development in recent years of macromolecular agents, such as targeted proteins, trophic factors, synthetic oligonucleotides, and genetic vectors, which have potential for therapeutic applications in various central nervous system (CNS) diseases. Among these strategies, antisense oligodeoxynucleotides (ODNs) have repeatedly demonstrated efficacy in reducing the expression of various gene products in a wide variety of cell types.[27,28] One of the initial obstacles in the use of antisense oligonucleotides in biological systems was the marked sensitivity of native phosphodiester ODNs to degradation by ubiquitous nucleases. A variety of chemical modifications have since been developed to render the native oligonucleotides more resistant to degradation, and the phosphorothioate modification of the oligonucleotide backbone has advanced farthest as a class of potential clinical drug candidates.[2,5,12] Although extensive experience has begun to accumulate with phosphorothioate (PS)-ODNs in cell culture systems and in other in vivo systems, $[2-4,15,16,26,33]$ very little work has been devoted to the application of antisense techniques in the CNS. Presumably, this is in large part due to the poor penetration of oligonucleotides through the blood-brain barrier after systemic administration.[25] Nevertheless, antisense oligonucleotide techniques have the advantage of being applicable to a wide variety of gene products whose mRNA nucleotide sequences are readily available in the scientific literature. For this reason, we believed that studying the feasibility of delivering antisense PS-ODN into the CNS by using a high-flow microinfusion technique was of great importance. We report here our initial studies on the distribution and disposition of ${ }^{35} \mathrm{~S}-\mathrm{PS}-\mathrm{ODN}$ in normal rat brain after high-flow microinfusion.

\section{MATERIALS AND METHODS}

\section{Synthesis of Phosphorothioate Oligonucleotide}

The 18-mer oligonucleotide, a hybrid oligonucleotide, contains the sequence 5'-AUG TTG CCG CAT CAU AAA-3'. Synthesis of the 18-mer was performed using deoxynucleoside phosphoramidites and 2'-O-methyl ribonucleoside phosphoramidites on an automated synthesizer (Biosearch 8800). The synthesis was performed on a 1-mmol scale by using the protocols previously reported.[22] The first three couplings were conducted using 2'-O- methyl ribonucleoside beta-cyanoethylphosphoramidite onto 2'-O-methyl ribonucleoside, a derivatized, controlled-pore glass support. After the first three couplings, the next 11 couplings were performed using deoxynucleoside beta-cyanoethylphosphoramidite. The last three couplings of the sequence were conducted using 2'-O-methyl ribonucleoside phosphoramidite. After each coupling, the linkage was oxidized with ${ }^{3} \mathrm{H}$-1,2-benzodithiol-3-one-1,1-dioxide to generate phosphorothioate linkage.[22] Deprotection and purification of the hybrid ODN were performed using the same procedures as reported earlier.[22] The purified ODN was analyzed by capillary gel electrophoresis and ${ }^{31} \mathrm{P}$ nuclear magnetic resonance imaging. The percentage purity of the 18 -mer in the lyophilized sample was $93.6 \%$ by capillary gel electrophoresis, with the remainder being $n-1$ and $n-2$ products. 


\section{Synthesis of ${ }^{35}$ S-Hybrid ODN}

To prepare the ${ }^{35} \mathrm{~S}$-hybrid ODN, synthesis was carried out in a manner similar to that described previously except that the last three couplings were carried out using 2'-O-methyl ribonucleoside $\mathrm{H}$-phosphonate. The 2'-O-methyl ribonucleoside $\mathrm{H}$-phosphonates, A, U, and G, were synthesized by following the $\mathrm{PCl}_{3} /$ triazole method,[14] starting from the appropriate 2'-O-methyl ribonucleoside and isolated as triethylammonium salts. The isolated 2'-O-methyl ribonucleoside $\mathrm{H}$-phosphonates, A, U, and $\mathrm{G}$, were analyzed by ${ }^{31} \mathrm{P}$ and ${ }^{1} \mathrm{H}$ nuclear magnetic resonance spectroscopy. Prior to their use in the ODN synthesis, the nucleoside H-phosphonates were evaporated to dryness twice with anhydrous pyridine and dissolved into anhydrous pyridine/acetonitrile (1:1) to a concentration of $40 \mathrm{mM}$. After the assembly, the controlled pore glass-bound oligonucleotide containing three $\mathrm{H}$-phosphonate linkages was oxidized with elemental ${ }^{35} \mathrm{~S}$ (Amersham, 0.5 to $2.5 \mathrm{Ci} /$ milliatom) and deprotected by the same procedure as reported earlier.[22] Purification of the ${ }^{35} \mathrm{~S}$-hybrid ODN was performed by using $20 \%$ polyacrylamide gel electrophosphoresis (containing 7M urea). The bands were excised under ultraviolet light, extracted in $100-\mathrm{mm}$ ammonium acetate, and desalted using a C18 column. The specific activity of the ODN obtained was $0.24 \mu \mathrm{Ci} / \mu \mathrm{g}$. Solutions of 18 -mer oligonucleotide were prepared in mock cerebrospinal fluid (CSF).

\section{Animal Experiments}

Twenty-five female Fischer 344 rats, each weighing between 150 and $160 \mathrm{~g}$, were anesthetized by using a combination of intraperitoneally administered ketamine $(50-80 \mathrm{mg} / \mathrm{kg})$ and xylazine $(5-10 \mathrm{mg} / \mathrm{kg})$ and were positioned in a stereotactic frame. A burr hole was made $3.5 \mathrm{~mm}$ lateral and $1 \mathrm{~mm}$ posterior to the bregma, and a 25-gauge needle was advanced to a depth of $4.5 \mathrm{~mm}$ to permit infusion into the caudate putamen.

Twenty microliters of a $100-\mu \mathrm{M}$ solution containing $0.5 \mu \mathrm{Ci}$ of the 18 -mer ${ }^{35} \mathrm{~S}-\mathrm{PS}-\mathrm{ODN}$ was infused over 1 hour at a rate of $0.3 \mu \mathrm{l} /$ minute. The rats were killed at 1, 6, 12,24, and 48 hours after the start of the infusion, with five rats in each time-point group. The brains were removed and snap-frozen in isopentane/dry ice and stored at $-80 \check{\mathrm{S} C}$ for cryosectioning.

Cryotome sections $(16 \mu \mathrm{m})$ were cut and used for quantitative autoradiography and extraction of oligonucleotide to study their stability by means of gel electrophoresis. Serial sections (four of every 20) of brain were applied to microscope slides, air dried, and then apposed to Kodak Biomax MR film for 18 to 36 hours. The x-ray films were developed and the resulting autoradiograms were analyzed by computer-assisted image analysis. Cryosections of a series of concentrations of $\left[{ }^{35} \mathrm{~S}\right]$ sulfate mixed in known amounts of homogenized brain were used as brain-paste standards and included in each autoradiogram.

Cerebrospinal fluid was collected from the cisterna magna of selected rats at 30 minutes and at 1, 2, 4, 6, 12 , and 24 hours after the start of the infusion. Samples were placed in vials for scintillation counting. The values for average ${ }^{35} \mathrm{~S}$-labeled oligonucleotide concentration and volumes of distribution were used to calculate the total activities within the brain parenchyma. The activities recovered in CSF (shown in Fig. 5, as well as in Table 2) were calculated by assuming the volume of CSF of $100 \mu \mathrm{l}$ in the rat. The resulting values of activity in the brain parenchyma and CSF for each time point were then summed to calculate the total activity recovered, as well as the percentages that these represent of the original infused activity $(0.5 \mu \mathrm{Ci})$. 


\section{Extraction and Analysis of Nucleic Acids From Brain and CSF Samples}

Brain sections obtained in parallel with those used for autoradiography were transferred to a prechilled mortar and pestle on dry ice and ground into powder. The powdered tissue was then suspended in digestion buffer $(2 \mathrm{mg} / \mathrm{ml}$ proteinase $\mathrm{K}, 0.5 \%$ sodium dodecyl sulfate, $10 \mathrm{mM} \mathrm{NaCl}, 20 \mathrm{mM}$ Tris-HCL-pH 7.6, $10 \mathrm{mM}$ ethylenediamine tetraacetic acid) using $1.2 \mathrm{ml} / 100 \mathrm{mg}$ of tissue. The sample was incubated at $37 \mathrm{~s} C$ for 2 hours, followed by two extractions with phenol/chloroform/isoamyl alcohol (25:24:1) and one extraction with chloroform. The 18-mer was then precipitated using ice-cold ethanol. The resulting pellet was dried, resuspended in water, and applied to a $20 \%$ polyacrylamide/7M urea gel for electrophoresis. Following electrophoresis, the gel was covered with plastic film and exposed to Kodak Biomax MR film for 24 to 48 hours to visualize the ${ }^{35} \mathrm{~S}$-material.

\section{Sources of Supplies and Equipment}

The 18-mer oligonucleotide was provided by Sudhir Agrawal, Ph.D., of Hybridon, Inc., Cambridge MA. The deoxynucleoside phosphoramidites were obtained from Millgen, Milford, MA; the 2'-O-methyl-ribonucleoside phosphoramidites from Glen Research, Sterling, VA; and the dry ice (2-methyl butane) and ethanol from Sigma Chemical Co., St. Louis, MO.

The Sep-Pak Column was acquired from Waters, Milford, MA; the stereotactic frame from David Kopf Instruments, Tujunga, CA; the Kodax Biomax MR film from Eastman Kodak Co., Rochester, NY; and the computer-assisted image analyzer (M4/MCID System) from Imaging Research, Inc., St. Catherines, ON, Canada.

\section{RESULTS}

\section{Distribution and Persistence of ${ }^{35}$ S-PS-ODN After Direct Intraparenchymal Infusion}

The pattern of distribution and persistence of ${ }^{35} \mathrm{~S}-18$-mer phosphorothioate oligonucleotide in rat brain is illustrated by the array of autoradiograms in Fig. 1. Each column of autoradiograms represents a sequential series of cryosections of rat brain taken at the times indicated after beginning infusion. The infusion rate was set at $0.3 \mu \mathrm{l} /$ minute. Previous work by Lieberman, et al.,[18] showed that infusion rates between 0.01 and $0.2 \mu \mathrm{l} /$ minute did not result in any retrograde flow along the needle tract; however, in larger species infusion rates with sequential increases up to $4 \mu \mathrm{l} /$ minute are well tolerated. We have shown that infusion rates in increments from 0.25 to $2 \mu \mathrm{l} /$ minute are well tolerated in rats with no retrograde flow along the needle tract (unpublished data). The column on the left demonstrates the normal histological appearance of the sections, which are arranged in anterior to posterior order (Fig. 1). Thus, sections taken through the caudate putamen demonstrated the needle tract as an area of intense focal activity. At the 1-hour time point, taken immediately following completion of the infusion, high concentrations of oligonucleotide were noted in a circumscribed area around the needle tract, with some additional extension of activity along adjacent white matter tracts. At subsequent time points, the activity was observed to spread radially into the surrounding brain, which is indicated by the wider area of distribution of sulfur-35 activity on each slice, as well as the greater anterior to posterior extent of the activity. 


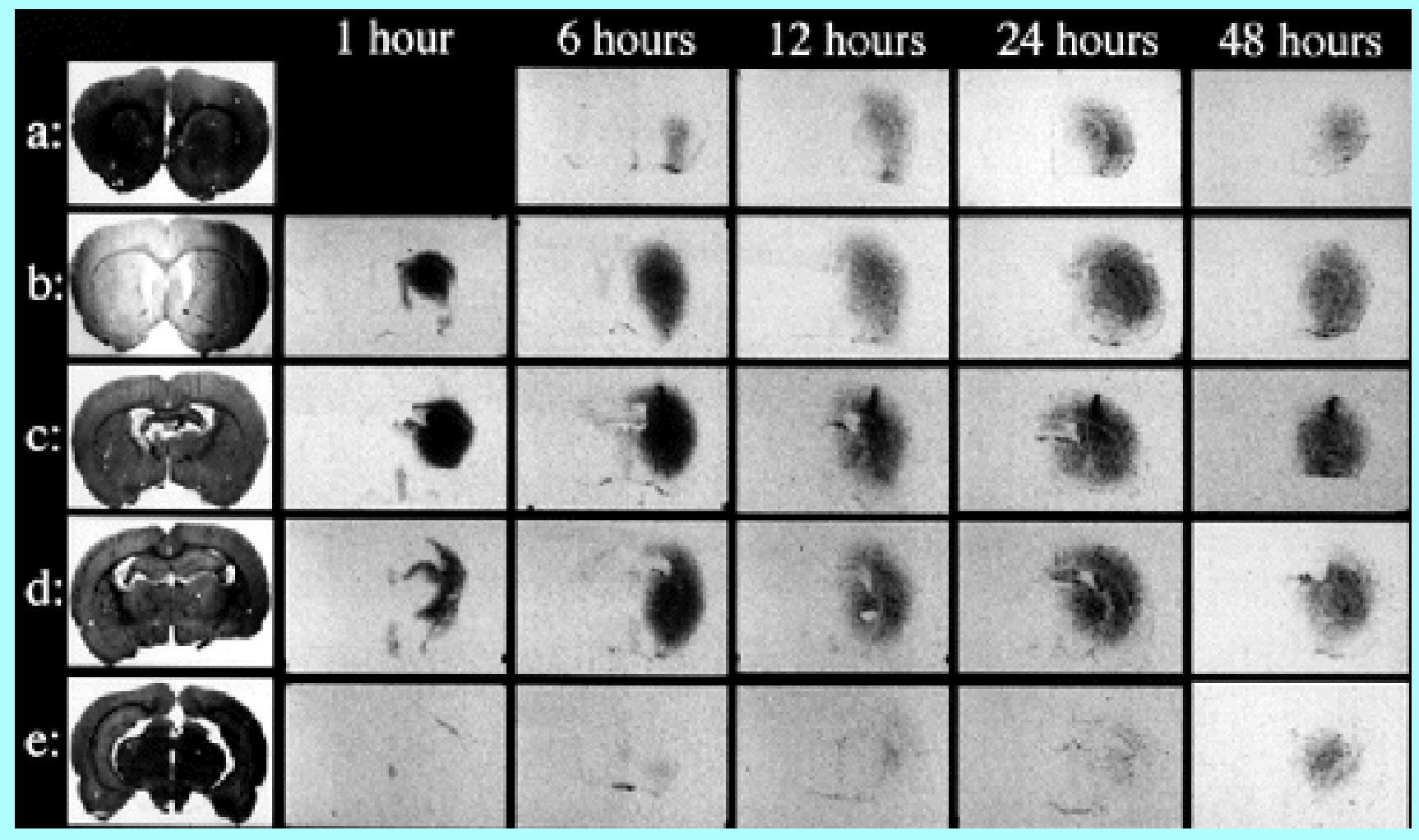

Fig. 1. Autoradiographic distribution of ${ }^{35}$ S-PS-ODN after infusion in rat brain representative coronal sections (far left column) and autoradiograms (all other columns) after high-flow microinfusion of $20 \mu \mathrm{l}$ of $100 \mathrm{mM}{ }^{35}$ S-PS-ODN. Because the infusion was carried out over a period of 1 hour, the first column (1 hour) of autoradiograms represents the status of distribution of ${ }^{35}$ S-PS-ODN immediately after completion of the infusion and subsequent time points represent 5, 11, 23, and 47 hours after completing the infusion, respectively. Rows a through e are arranged from anterior to posterior, and the location of the sections were as follows: a) $1.70 \mathrm{~mm}$ anterior to bregma; b) at bregma; c) $1 \mathrm{~mm}$ posterior to bregma-point of infusion; d) $1.80 \mathrm{~mm}$ posterior to bregma; and e) $5.80 \mathrm{~mm}$ posterior to bregma.

Each set of autoradiograms was obtained in conjunction with cryosections of brain-paste standards containing known quantities of ${ }^{35} \mathrm{~S}-\mathrm{PS}-\mathrm{ODN}$. This allowed quantitative analysis of the distribution of oligonucleotide at selected time points following completion of the infusion. Fig. 2 provides one representation of this analysis: each point represents the average density of oligonucleotide within its area of distribution on groups of consecutive sections arranged in anterior to posterior order. The resulting curves demonstrate the high focal concentrations attained immediately at the end of the infusion process and the subsequent anteroposterior spread of the material within the brain, with concomitant decreases in tissue concentration in the hours following completion of the infusion. Even at 48 hours, however, the resulting tissue concentrations of oligonucleotide were significant, as will be discussed. 


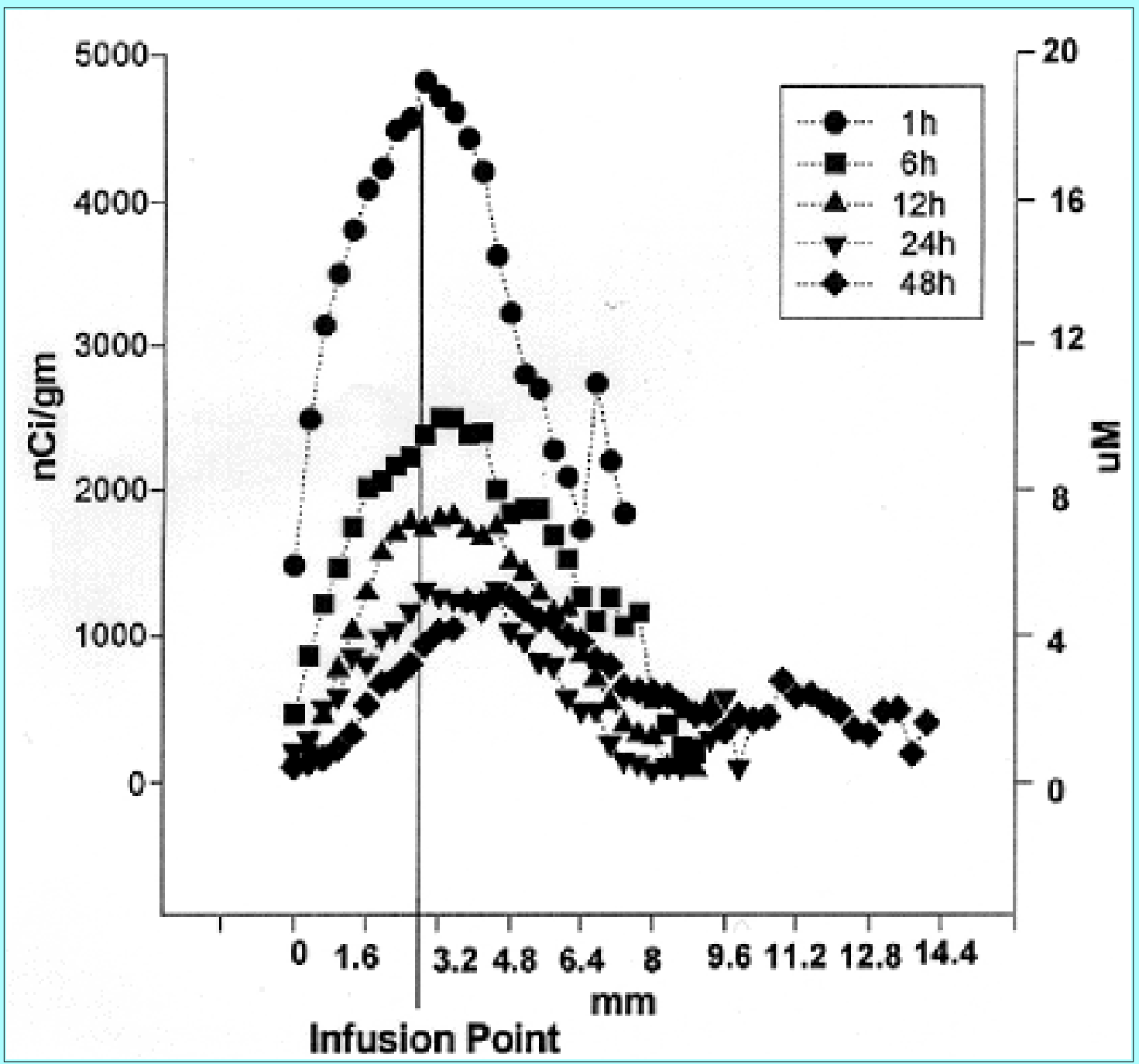

Fig. 2. Graph showing quantitative distribution of ${ }^{35}$ S-PS-ODN after infusion in rat brain. The anterior to posterior profiles of ${ }^{35} \mathrm{~S}-\mathrm{PS}-\mathrm{ODN}$ tissue content in rat brain after high flow microinfusion are shown, and the time points correspond to those depicted in Fig. 1. The values shown were obtained by quantitative autoradiographic technique using computerized image analysis. A region of interest was delineated to surround the region of distribution of sulfur-35 activity, and the average activity was calculated from the relative optical density using brain-paste standards. The values shown represent the averages of comparable sections taken from multiple animals. Standard deviation values were in the range of $15-25 \%$ (error bars not shown for purposes of clarity).

Using imaging analysis techniques, the areas of distribution for each group of consecutive sections and the thickness of the cryosections were used to calculate volumes of distribution of oligonucleotide at each time point. The mean values ( \pm standard deviations) of this parameter for the groups of animals at each time point are shown in Table 1 . Immediately following the $20-\mu \mathrm{l}$ infusion, the volume of distribution was $105 \pm 7.9 \mu$, representing a ratio of $\mathrm{V}_{\mathrm{d}} / \mathrm{V}_{\mathrm{i}}$ of 5.2. The volume of distribution was seen to increase substantially in the ensuing hours, to a value of $443 \pm 62.3 \mu \mathrm{l}$ at 48 hours. As would be expected, the tissue content of ${ }^{35} \mathrm{~S}-\mathrm{PS}-\mathrm{ODN}$ decreased commensurately over the same time period. These values are also shown in Table 1, in conjunction with minimum tissue concentrations corresponding to the values for tissue content. This concentration was calculated by converting tissue content of ${ }^{35} \mathrm{~S}-\mathrm{PS}-\mathrm{ODN}$ in microcuries per gram to micromoles per liter using the specific activity of infused 
${ }^{35} \mathrm{~S}-\mathrm{PS}-\mathrm{ODN}$ and estimating the specific gravity of brain to be approximately $1 \mathrm{~g} / \mathrm{ml}$. This minimum concentration would assume that the ${ }^{35} \mathrm{~S}$ oligonucleotide was distributed uniformly within the tissue slice without regard to the fractional volumes of intra- versus extracellular space. If the fractional volume of edematous extracellular space is assumed to be 10 to $40 \%,[6,7]$ and if the oligonucleotide is assumed to be distributed only within the extracellular space, the actual interstitial concentration value could be increased by a factor of 2.5 to 10 . Thus, direct intraparenchymal infusion of $35 \mathrm{~S}$-PS-ODN allows for wide distribution and persistence of concentrations of oligonucleotide greater than those reported to have significant effects in in vitro systems $[1,10,18](3-10 \mu \mathrm{M})$.

\begin{tabular}{|c|c|c|c|}
\hline \multirow[b]{3}{*}{$\begin{array}{l}\text { Postinf usion } \\
\text { Time (hrs) }\end{array}$} & \multirow[b]{3}{*}{$\begin{array}{c}\text { Yolume of } \\
\text { Distribution }(\mu)]\end{array}$} & $\begin{array}{l}\text { E } 1 \\
\text { TISSUE CONTEN } \\
\text { PARENCHYMAL }\end{array}$ & $\begin{array}{l}35 \mathrm{~S}-\mathrm{PS}-\mathrm{ODN} \\
\text { ISIN* }\end{array}$ \\
\hline & & \multicolumn{2}{|c|}{ A Awerage 35 S-PS-CDN Corkent } \\
\hline & & $\begin{array}{c}\text { Tissue Corkert } \\
\left(\mu \mathrm{Cir}^{\prime} \mathrm{g}\right]\end{array}$ & $\begin{array}{l}\text { Mirim un Tissue } \\
\text { Concerkration } \\
{[\mu \text { ollit }] \dagger}\end{array}$ \\
\hline $\begin{array}{r}1 \\
6 \\
12 \\
24 \\
48\end{array}$ & $\begin{array}{l}105 \pm 7.9 \\
173 \pm 26.5 \\
268 \pm 12.2 \\
289 \pm 36.1 \\
443 \pm 62.3\end{array}$ & $\begin{aligned} 3.8 & \pm 0.3 \\
2 & \pm 0.4 \\
1.3 & \pm 0.14 \\
1 & \pm 0.2 \\
0.8 & \pm 0.14\end{aligned}$ & $\begin{array}{c}15.2 \\
8 \\
5.2 \\
4 \\
3.2\end{array}$ \\
\hline \multicolumn{4}{|c|}{$\begin{array}{l}\text { * Values expressed as the mean } \pm \text { standard deviation. } \\
\dagger \text { Calc uated from tissue contert by using specific activity of } 3_{\mathrm{S}-\mathrm{PS}-\mathrm{ODN} \text { and }} \\
\text { assuming the specific gravity of the brain to be approximately } 1 \mathrm{~g} \text { 'ml. }\end{array}$} \\
\hline
\end{tabular}

\section{Stability of ${ }^{35}$ S-PS-ODN After Direct Intraparenchymal Infusion}

To determine the stability of the modified phosphorothioate 18-mer oligonucleotide after infusion into rat brain, sections adjacent to those used for autoradiography were subjected to solubilization and extraction of nucleic acids. Following ethanol precipitation, the nucleic acids were redissolved in aqueous buffer and applied to a $20 \%$ polyacrylamide/7M urea gel for electrophoresis. Figure 3 demonstrates the autoradiogram from such a gel. A single band from each time point sampled can be seen to comigrate with a sample of stock 18 -mer ${ }^{35}$ S-PS-ODN (note that the quantity of material in the control lane was selected arbitrarily and is not intended to be compared in intensity to the amount extracted from the brain slices). Degradation of the 18-mer by nucleases would be expected to yield a series of smaller oligonucleotides, resulting in bands or a smear of activity with greater electrophoretic mobility below that of the 18-mer. At 48 hours, a slight degree of degradation was represented by some smearing of the sulfur-35 activity band. Thus, even after 48 hours in the brain parenchyma, the majority of sulfur-35 activity retained the electrophoretic mobility characteristic of undegraded 18-mer PS-ODN. 


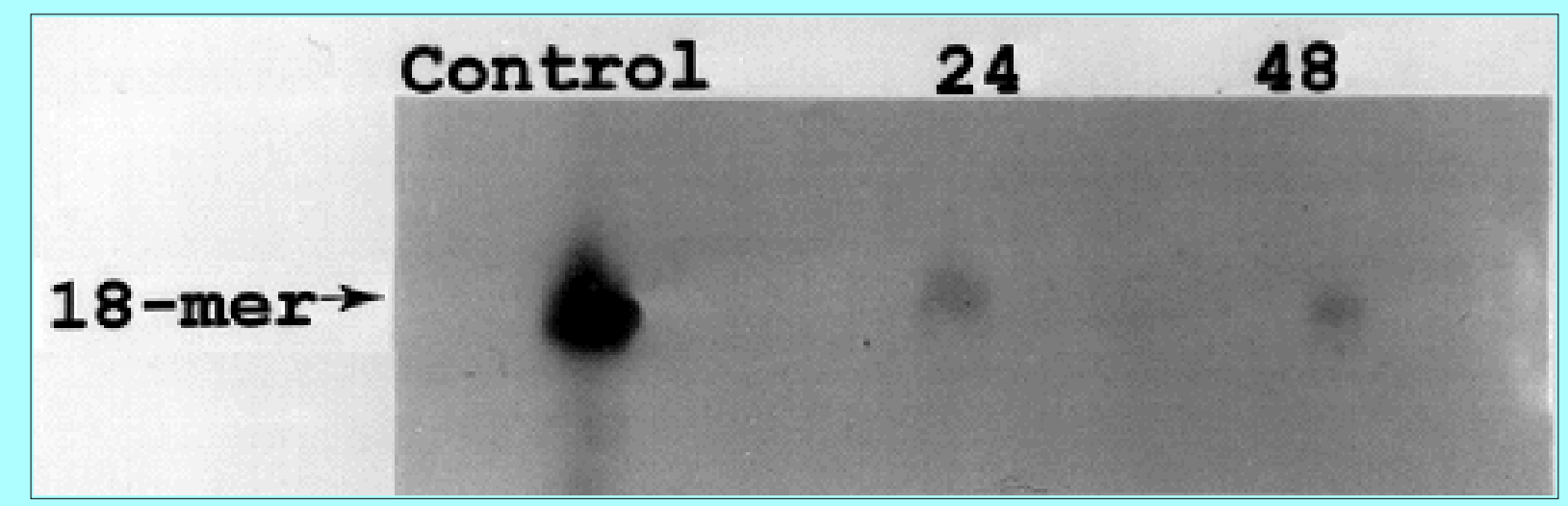

Fig. 3. An autoradiogram of a $20 \%$ polyacrylamide/7M urea gel showing stability of ${ }^{35}$ S-PS-ODN 18-mer after direct parenchymal infusion. Extracts of brain sections obtained at the times indicated were subjected to electrophoresis. The large majority of oligonucleotide present is intact 18-mer. At the latest time point, faint activity at higher mobilities can be seen, indicating minimal degradation of the 18-mer to lower molecular-weight nucleotides.

\section{Distribution and Clearance Within CSF of ${ }^{35}$ S-PS-ODN After Direct Intraparenchymal Infusion}

Careful inspection of the 1-hour autoradiograms in Figure 1, obtained immediately at the end of the infusion, demonstrated the presence of significant levels of sulfur-35 activity within the ventricular system. The upper image in Fig. 4 confirms this as well, indicating the presence of ${ }^{35}$ S-PS-ODN within ventricular CSF immediately following completion of infusion. Quantitative autoradiography indicated that these levels represent a concentration of $12.4 \pm 4.6 \mu \mathrm{M}$, a level that compares well with the quantity of sulfur-35 activity obtained from cisterna magna CSF samples discussed later. A representative autoradiogram obtained from an animal 6 hours after the beginning of an infusion demonstrated a substantial decrease in sulfur-35 activity within the CSF space itself (Fig. 4 center). At this time point, however, substantial sulfur-35 activity can be seen to be associated with the ependymal and periventricular zone. A representative autoradiograph obtained from an animal killed at 24 hours (Fig. 4 lower) revealed dissipation of the enhanced periventricular activity. 


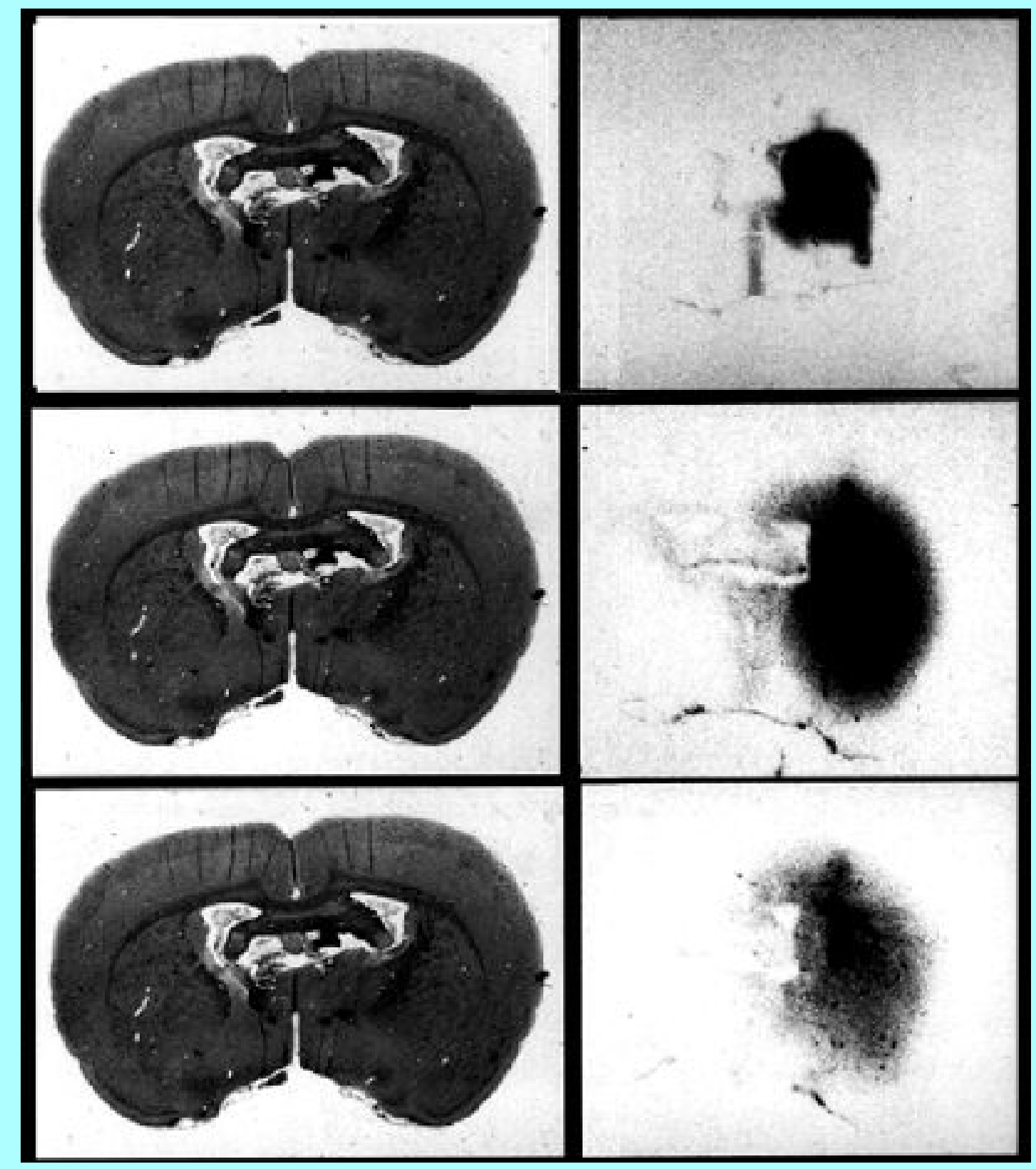

Fig. 4. Selected autoradiograms and comparable brain sections in animals sacrificed at 1, 6, and 24 hours after starting the infusion. Upper: Note the presence of sulfur-35 activity in the CSF space of the ventricles and interhemispheric fissure in the 1-hour specimen taken immediately after completion of the infusion. Center: By 6 hours, the CSF space had substantially cleared, but significant activity is noted in the ependyma and ependymal zone of the ventricular system. Lower: By 24 hours, the enhanced activity of the periventricular region is also lost, and the activity is more homogenous with the contiguous spread of activity from the infusion site.

The penetration of ${ }^{35} \mathrm{~S}$-PS-ODN into the CSF space during the infusion process was confirmed by studies monitoring the appearance of sulfur-35 activity in cisterna magna CSF samples (Fig. 5). A rapid rise of ventricular sulfur-35 activity occurred during the infusion process, reaching a maximum value that represented approximately 10\% (50 $\pm 20 \mathrm{nCi})$ of the infused activity. Thereafter, a rapid exponential decay of activity within the CSF was noted in the ensuing 24 hours. Between 24 and 48 hours, the activity appearing in CSF stabilized at a low but finite level. A semi-logarithmic plot of this decay curve demonstrated three phases of the CSF distribution and clearance process. These included: 1) the infusion 
phase (30 and 60 minutes), with rapidly increasing content of ${ }^{35}$ S-PS-ODN in CSF; 2) a first-order decrease in CSF concentration between 1 and 12 hours; and 3) a basal efflux phase after 12 hours that is characterized by a steady-state concentration of ${ }^{35}$ S-oligonucleotide in CSF. The identity of sulfur-35 activity in CSF as undegraded 18 -mer ${ }^{35}$ S-PS-ODN was confirmed by submitting samples of CSF to $20 \%$ polyacrylamide/7M urea gel electrophoresis (data not shown), as described previously, for studies of stability of intraparenchymal ${ }^{35}$ S-PS-ODN.

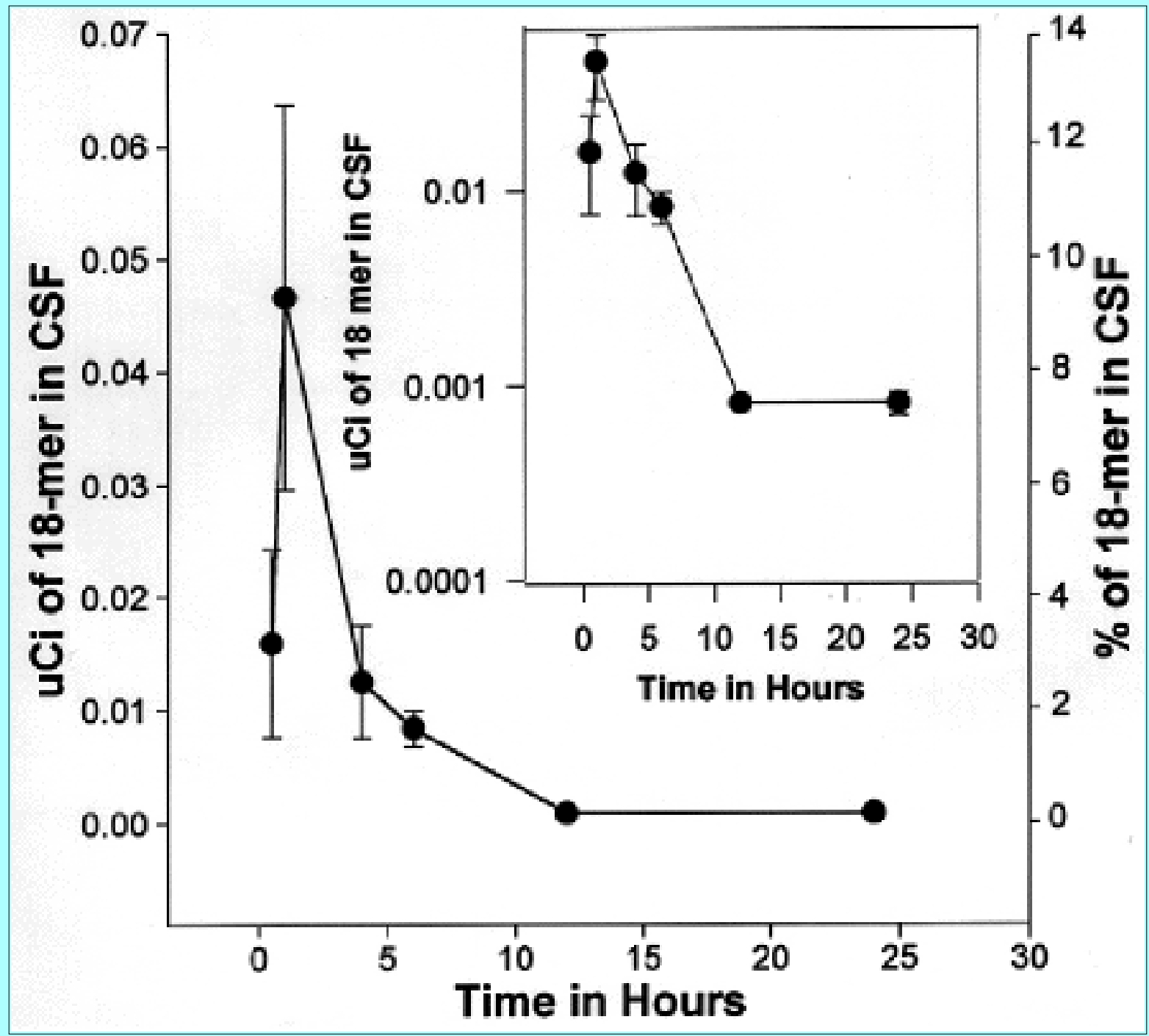

Fig. 5. Graph illustrating the appearance of ${ }^{35}$ S-PS-ODN in samples of direct intraparenchymal-infusion cisterna magnum CSF. Scintillation counting allowed for calculation of activity in the CSF space, which is shown on the left. The percentage of infused activity which this represents is indicated on the right vertical axis. Substantial activity is noted at 30 minutes, and this value increases to a maximum at 1 hour, corresponding to the time when infusion was completed. Thereafter, an exponential decrease in CSF sulfur-35 activity is noted. When these data are plotted on semilogarithmic scale, the decrease in activity is noted to have two components: an exponential component and a steady-state component. For further discussion, see text.

\section{Recovery of Infused Activity}

Table 2 summarizes the activity measurements recovered from brain parenchyma and CSF at each indicated time point. After the 1-hour time point, representing the time of completion of the infusion, 
nearly all of the infused activity was accounted for by that found in parenchyma and CSF. This value dropped slowly over ensuing hours and days and fell only to approximately $70 \%$ by 48 hours.

\begin{tabular}{|c|c|c|c|}
\hline CALCULATIOI & 35 S-ODN RECON & $\begin{array}{l}2 \\
\text { RAT BRAN F }\end{array}$ & NCHYMA AND CSF* \\
\hline $\begin{array}{l}\text { Postinf usion } \\
\text { Time (hrs) }\end{array}$ & $\begin{array}{c}\text { Activity in } \\
\text { Par enc tyma }(\mu \mathrm{Ci})\end{array}$ & $\begin{array}{l}A c-b i v i t y \text { in } \\
\operatorname{CSF}(\mu \mathrm{Ci})\end{array}$ & $\begin{array}{c}\text { Percentage of } \\
\text { Total Infused } \\
\text { Activity Rec overed }\end{array}$ \\
\hline $\begin{array}{r}1 \\
6 \\
12 \\
24 \\
48\end{array}$ & $\begin{array}{l}0.39 \pm 0.03 \\
0.34 \pm 0.06 \\
0.34 \pm 0.03 \\
0.28 \pm 0.04 \\
0.35 \pm 0.06\end{array}$ & $\begin{array}{c}0.05 \pm 0.02 \\
0.01 \pm 0 \\
0 \pm 0 \\
0 \pm 0 \\
0 \pm 0\end{array}$ & $\begin{array}{l}88 \pm 10 \\
70 \pm 12 \\
68 \pm 6 \\
56 \pm 8 \\
70 \pm 12\end{array}$ \\
\hline - & do the man & nad-ointin & \\
\hline
\end{tabular}

\section{Intraparenchymal Flow Patterns of ${ }^{35}$ S-PS-ODN After Direct Infusion}

The pattern of distribution of sulfur-35 activity shown in the autoradiogram of Fig. 1 provides evidence for differential characteristics of distribution of infusate between structures containing predominantly white matter and those containing predominantly gray matter (Fig. 1 rows b and c). Immediately after completing infusion, the 1-hour time point demonstrated radial distribution of intense activity into the caudate putamen, but significant activity was also noted in the overlying white matter extensions of the corpus callosum. This latter feature is also well demonstrated in Fig. 4 upper. By 6 hours the activity had spread beyond the white matter tract and began to enter the cerebral cortex overlying it. This trend continued at 12 hours, and by 24 hours the activity was noted to have spread to the cortical surface. By 48 hours, the distribution of activity had receded from the cortical surface slightly, suggesting loss of ${ }^{35}$ S-PS-ODN directly into the CSF of the adjacent subarachnoid space.

Certain features of the behavior of sulfur-35 activity in the white matter tract overlying the caudate putamen supported the concept of increased interstitial fluid transport within this structure. The hyperintensity of activities seen in this structure on the autoradiograms obtained at 1 hour was noted to be isointense by 6 and 12 hours. By 24 and 48 hours, hypointensity was noted within this structure on multiple sections (see Fig. 1). Nevertheless, despite apparent differences in the rate of ${ }^{35}$ S-PS-ODN distribution in these different structures, widespread distribution in both white- and gray-matter structures can be obtained with the high-flow microinfusion technique.

\section{DISCUSSION}

High-flow microinfusion is a novel technique for delivering macromolecular agents to widespread areas of brain parenchyma. The feasibility of using this technique to deliver proteins with therapeutic potential into the brain has been demonstrated in feline, primate, and rodent models, $[9,18]$ and our laboratory has confirmed the viability of this technique in a rat model using macromolecular dye solutions. [24] The biophysical mechanisms by which this process occurs are not fully understood; however, it would appear that it is quite similar to the bulk flow of fluids that occurs normally in brain tissue and accounts for distribution of edema fluids.[20,21,29] This process can be characterized as a convective flow in which the driving force is a pressure gradient, as opposed to the process of diffusion which is driven by a concentration gradient. A salient feature of this difference is that the process of bulk flow allows for substantially wider distribution of solute molecules included in the infusate, and this has great potential for the delivery of therapeutic agents that would otherwise be excluded by the blood-brain barrier. Our 
current results are the first to demonstrate the feasibility of using high-flow microinfusion to deliver modified oligonucleotides in a similar fashion. These findings are particularly significant because of the wide variety of potential applications that the antisense approach allows. Our laboratory is currently investigating the potential of c-myc antisense PS-ODNs to inhibit tumor growth and prolong survival in a rat model of malignant glioma,[11] but other neuropathological processes that may involve excessive or inappropriate expression of cellular gene products would also be amenable to antisense oligonucleotide therapy using this delivery technique.

Using a single set of infusion parameters (volume, rate, and concentration of oligonucleotide), we have confirmed that high concentrations of oligonucleotide can be delivered to a volume of distribution more than fivefold greater than the volume infused. Furthermore, substantial oligonucleotide levels are maintained for hours following the infusion. These levels are greater than those needed for effects in vitro, when PS-ODNs are added to the media of cultured cells[1,10,18] $(3-10 \mu \mathrm{M})$.

To date, little work has been reported on the application of antisense oligonucleotide techniques in the CNS, presumably due to their very poor penetration of the blood-brain barrier. Whitesell, et al.,[31] have characterized the infusion of oligonucleotide concentrations into CSF via the lateral ventricle but did not report on the intraparenchymal concentrations attained using this technique. In light of the work by Blasberg, et al.,[8] it is likely that this penetration was quite low; they have shown that in primates with ventriculocisternal perfusions of low-molecular-weight chemotherapeutic agents penetration into the brain from ventricular fluid was extremely limited. They demonstrated a 2.5 - to 10 -fold reduction in concentration of chemotherapeutic agent as little as $1 \mathrm{~mm}$ from the ventricular surface. The work presented here stands in stark contrast to these findings and demonstrates the value of high-flow microinfusion technique in delivering macromolecular agents with therapeutic potential into the interstitial space by bypassing the blood-brain barrier.

Extraction of the 18-mer oligonucleotide after prolonged exposure to brain parenchyma followed by gel electrophoresis indicates marked stability of this particular oligonucleotide under these circumstances. This is attributable in part to the enhanced stability of the phosphorothioate modification, as well as to the 2'-O-methyl derivatization of selected nucleotides on the 5' and 3' ends of the sequence.[2] It would thus appear that appropriate experimental testing and oligonucleotide design can identify modified antisense oligonucleotides that can be widely delivered and remain stable in the brain parenchyma for prolonged periods of time. A great deal of work on the effects of antisense oligonucleotides and biological systems has identified a variety of other potential drawbacks to this approach. Some, such as the short duration of phosphodiester oligonucleotides activity and the possibility of nonspecific effects, are now routinely dealt with in the development of antisense strategies. Thus, phosphorothiote modifications render the oligonucleotides sustantially more stable in biological systems, and appropriate selection and testing of their sequences allows avoidance of nonspecific effects. Other potential drawbacks, particularly to CNS applications, remain to be addressed. These include the possibility of toxicity of the compounds after long-term exposure, such as might be used under therapeutic circumstances. Another major issue that may be significant is the limited access of oligonucleotides to their intracellular sites of action, but a variety of strategies under development in this area show great promise in dealing with this obstacle for therapeutic applications. Indeed, it is likely that the micromolar concentrations used for biological effects of phosphorothioate oligonucleotides in vitro reflect the fact that concentration orders of lower magnitude are needed intracellularly to attain their effects. We have been able to obtain interstitial levels of oligonucleotides that appear to be comparable to or greater than the levels needed in vitro for the inhibition of c-Myc expression. This is quite promising, particularly in 
view of the parallels between infusions that may remain predominantly in the extracellular space in vivo as well as the addition of antisense oligonucleotides to tissue-culture media that also represents the "extracellular space" in vitro.

We believe the early appearance of infused oligonucleotide in ventricular CSF and the apparent flow of infused material into the white matter tract overlying the caudate putamen are highly significant and related features of the infusion process. Previous histological studies of rat brain after infusion of small volumes $(3-10 \mu \mathrm{l})$ have demonstrated microscopic structural changes in these same white matter tracts.[24] These findings suggest that microstructural changes in brain parenchyma are an early component of the process that allows the widespread travel of infused solutions into the brain parenchyma. Our finding of early entry into ventricular CSF suggests that the white matter tract serves as a conduit for preferential flow of infusate. Weller, et al.,[30] and Zhang, et al.,[32] have shown in their rodent studies that white matter tracts act as conduits for the clearance of infusate into the ventricles. The white matter tracts can be seen to extend their fibers to a place very close to the ependymal surface of the lateral ventricles, where the entry of infusate into the CSF may take place. We would predict that the early entry of infusate into the ventricular CSF would be less significant in larger brains in which the distances to the ependymal surface are greater. Nevertheless, this phenomenon serves as an indicator of the importance of better understanding the biophysical mechanisms and microstructural changes underlying the distribution of infusate during and after high-flow microinfusion. Excellent work performed along these lines by Oldfield and collaborators, $[9,18]$ has begun to characterize such differences in infusion distribution kinetics in feline and primate brains. Although these differences represent potential complicating factors in the ability of high-flow microinfusion to deliver therapeutic agents predictably into human brain, we believe it will be possible to ensure adequate delivery into a variety of brain structures by better understanding these characteristics and appropriately placing the infusion sites. A limited confirmation of this concept is provided by the successful distribution of infused oligonucleotide through white matter into the gray matter in the rat cerebral cortex in studies reported here. Calculation of the recovered activity shown in Table 2 demonstrates that approximately $90 \%$ of the infused activity can be acounted for immediately on completion of the infusion. Although some of the losses could occur by backflow along the needle tract during the infusion process, we believe this to be a small component, because the infusion rate was selected specifically to avoid this complication. We have not attempted a complete evaluation of the biodistribution of oligonucleotides infused into the brain in this current work, as this would require obtaining blood samples and other tissue samples for measurement and pharmacokinetic analysis. Nevertheless, it is clear that even as long as 48 hours after the infusion, the majority of infused material remains in the brain parenchyma in its original, undegraded form.

Although the intent of these studies was not focused on toxicity, we nonetheless discovered no evidence of toxicity under these conditions. There were no behavioral signs of neurotoxicity in any of the animals up to 48 hours following infusion. Similarly, hematoxylin and eosin-stained sections of representative brains up to 48 hours after infusion of oligonucleotide also failed to show evidence of toxicity in the parenchyma. These results compare well with those reported by Whitesell, et al.,[31] who demonstrated global CNS depression and death in rats with intrathecal infusions of 15-mer phosphorothioate oligonucleotide at $1 \mu \mathrm{l} /$ minute, when the PS-ODN concentration exceeded $3 \mathrm{mM}$. The concentration of infusate in our studies was 30-fold lower than this threshold level and was infused at a lower rate for a shorter time period. Thus, our lack of evidence of toxic effects is not inconsistent with those studies.

Chiasson, et al.,[13] have demonstrated neurotoxic damage locally at the site of injection following 
repeated daily injections of $2 \mu \mathrm{l}$ of $1 \mathrm{mM} \mathrm{15-mer} \mathrm{phosphodiester} \mathrm{oligonucleotide} \mathrm{into} \mathrm{the} \mathrm{amygdala} \mathrm{of}$ rats. They concluded that the observed toxicity was greatly diminished when the time interval between the injections was extended. Whereas the dose of phosphodiester ODN infused was equivalent to the dose of PS-ODN used in our studies, the volume of distribution would have been expected to be much smaller, resulting in higher local concentrations of ODN. Furthermore, it is likely that the phosphodiester ODN would have been rapidly degraded to component nucleotides, the effects of which (at high local concentrations) would be unclear. Although no evidence of toxic effects has been noted in our studies thus far, more extensive studies on the toxicity of PS-ODN after high-flow microinfusion are clearly required.

This report represents our first use of this experimental system to study the pharmacokinetics of modified oligonucleotides delivered by high-flow microinfusion. The use of uniformly ${ }^{35} \mathrm{~S}$-phosphorothioate oligonucleotide in conjunction with quantitative autoradiographic techniques has made it possible to obtain extensive quantitative data on the distribution and stability of infused phosphorothioate oligonucleotide. This experimental system has several features that make it particularly useful for further studies and biophysical modeling of the high-flow microinfusion process. First, the use of ${ }^{35}$ S-oligonucleotide allows for rapid completion of the autoradiographic technique. The availability of uniformly labeled ${ }^{35}$ S-PS-ODN allows for more complete accounting for the status of the infused material, when compared with end-labeled or fluorescently labeled ODN techniques. Finally, the chemistry of oligonucleotides in biological systems allows for their extraction and electrophoretic characterization with relative ease. Indeed, it would also be feasible to subject the extracted material to bioassay as an ultimate test of the intactness of the infused oligonucleotide.

One additional advantage of this model system is the potential for extending these studies to the application of antisense oligonucleotide techniques in rodent models of neuropathological processes. We have demonstrated the efficacy of c-myc antisense phosphorothioate oligonucleotide to inhibit tumor growth in a rat model of intracerebral malignant glioma.[11] The availability of rat models of traumatic brain injury, ischemic injury, and neurodegenerative disorders raises the potential for studying antisense oligonucleotide strategies in these pathologies as well.

It is also clear that studies similar to that described here will need to be extended to models with larger brains. Along these lines, it is notable that a Phase I study of high-flow microinfusion for the delivery of a protein-toxin conjugate in patients with recurrent malignant brain tumors has been completed (D. Laske, personal communication), and a multicenter Phase II trial is underway. Thus, experience with high-flow microinfusion of protein solutions in human brain will provide important experience to help guide the development of this technique for the delivery of other agents.

In summary, high-flow microinfusion allows widespread delivery of modified antisense oligonucleotides into the brain. These materials demonstrate excellent stability and persistence following infusion. These findings suggest that clinical applications of antisense oligonucleotide techniques in the CNS have great potential.

\section{Acknowledgments}

The authors wish to acknowledge the generous provision of oligonucleotide and valuable comments and advice by Dr. Sudhir Agrawal and thank Drs. M. Ross Bullock and Xiao Di for their assistance and advice. 


\section{References}

1. Agrawal S, Ikeuchi T, Sun D, et al: Inhibition of human immunodeficiency virus in early infected and chronically infected cells by antisense oligodeoxynucleotides and their phosphorothioate analogues. Proc Natl Acad Sci USA 86:7790-7794, 1989

2. Agrawal S, Iyer RP: Modified oligonucleotides as therapeutic and diagnostic agents. Curr Opin Biotechnol 6:12-19, 1995

3. Agrawal S, Temsamani J, Tang JY: Pharmacokinetics, biodistribution, and stability of oligodeoxynucleotide phosphorothioates in mice. Proc Natl Acad Sci USA 88:7595-7599, 1991

4. Agrawal S, Zhang X, Lu Z, et al: Absorption, tissue distribution and in vivo stability in rats of a hybrid antisense oligonucleotide following oral administration. Biochem Pharmacol 50:571-576, 1995

5. Bacon TA, Morvan F, Rayner B, et al: alpha-Oligodeoxynucleotide stability in serum, subcellular extracts and culture media. J Biochem Biophys Meth 16:311-318, 1988

6. Bakay L: The extracellular space in brain tumours. I. Morphological considerations. Brain 93:693-698, 1970

7. Bakay L: The extracellular space in brain tumours. II. The sucrose space. Brain 93:699-708, 1970

8. Blasberg RG, Patlak C, Fenstermacher JD: Intrathecal chemotherapy: brain tissue profiles after ventriculocisternal perfusion. J Pharmacol Exp Ther 195:73-83, 1975

9. Bobo RH, Laske DW, Akbasak A, et al: Convection-enhanced delivery of macromolecules in the brain. Proc Natl Acad Sci USA 91:2076-2080, 1994

10. Broaddus WC, Chen ZJ, Prabhu SS, et al: Antiproliferative effect of c-myc antisense phosphorothioate oligodeoxynucleotide in malignant glioma cells. Neurosurgery 41:908-915, 1997

11. Broaddus WC, Prabhu SS, Chen ZJ, et al: High-flow microinfusion of c-myc antisense oligonucleotides prolongs survival in an animal model of intracranial malignant glioma. Neurosurgery 39:647, 1996 (Abstract)

12. Campbell JM, Bacon TA, Wickstrom E: Oligodeoxynucleoside phosphorothioate stability in subcellular extracts, culture media, sera and cerebrospinal fluid. J Biochem Biophys Meth 20:259-267, 1990

13. Chiasson BJ, Armstrong JN, Hooper ML, et al: The application of antisense oligonucleotide technology to the brain: some pitfalls. Cell Mol Neurobiol 14:507-521, 1994

14. Froehler BC: Oligodeoxynucleotide synthesis. H-phosphonate approach. Meth Mol Biol 20:63-80, 1993

15. Goodarzi G, Watabe M, Watabe K: Organ distribution and stability of phosphorothioated oligodeoxyribonucleotides in mice. Biopharm Drug Dispos 13:221-227, 1992

16. Iversen PL, Mata J, Tracewell WG, et al: Pharmacokinetics of an antisense phosphorothioate oligodeoxynucleotide against rev from human immunodeficiency virus type 1 in the adult male rat 
following single injections and continuous infusion. Antisense Res Dev 4:43-52, 1994

17. Jain RK: Transport of molecules in the tumor interstitium: a review. Cancer Res 47:3039-3051, 1987

18. Lieberman DM, Laske DW, Morrison PF, et al: Convection-enhanced distribution of large molecules in gray matter during interstitial drug infusion. J Neurosurg 82:1021-1029, 1995

19. Maltese JY, Sharma HW, Vassilev L, et al: Sequence context of antisense RelA/NF-kappa-B phosphorothioates determines specificity. Nucleic Acids Res 23:1146-1151, 1995

20. Marmarou A, Hochwald G, Nakamura T, et al: Brain edema resolution by CSF pathways and brain vasculature in rats. Am J Physiol 36:H514-H520, 1994

21. Marmarou A, Tanaka K, Shulman K: The brain response to infusion edema, in Hartmann A, Brock M (eds): Treatment of Cerebral Edema. New York: Springer-Verlag, 1982, pp 11-18

22. Metelev V, Lisziewicz I, Agrawal S: Study of antisense oligonucleotide phosphorothioates containing segments of oligodeoxynucleotides and 2'-O-methyl-oligoribonucleotides. Bioorg Med Chem Lett 4:2929-2934, 1994

23. Morrison PF, Laske DW, Bobo H, et al: High-flow microinfusion: tissue penetration and pharmacodynamics. Am J Physiol 266:R292-R305, 1994

24. Prabhu SS, Broaddus WC, Gillies GT, et al: Distribution of macromolecular dyes in brain using positive pressure infusion: a model for direct controlled delivery of therapeutic agents. Surg Neurol (In press, 1997)

25. Rapoport SI: Blood-Brain Barrier in Physiology and Medicine. New York: Raven Press, 1976, pp 99-111

26. Saijo Y, Perlaky L, Wang H, et al: Pharmacokinetics, tissue distribution, and stability of antisense oligodeoxynucleotide phosphorothioate ISIS 3466 in mice. Oncol Res 6:243-249, 1994

27. Stein CA, Cheng YC: Antisense oligonucleotides as therapeutic agents--is the bullet really magical? Science 261:1004-1012, 1993

28. Wagner RW, Matteucci MD, Lewis JG, et al: Antisense gene inhibition by oligonucleotides containing C-5 propyne pyrimidines. Science 260:1510-1513, 1993

29. Walstra G, Takagi H, Marmarou A, et al: The time course of brain tissue compliance and resistance in a controlled model of cerebral edema, in Shulman K, Marmarou A, Miller JD, et al (eds): Intracranial Pressure IV. New York: Springer-Verlag, 1980, pp 85-87

30. Weller RO, Kida S, Zhang ET: Pathways of fluid drainage from the brain--morphological aspects and immunological significance in rat and man. Brain Pathol 2:277-284, 1992

31. Whitesell L, Geselowitz D, Chavany C, et al: Stability, clearance, and disposition of intraventricularly administered oligodeoxynucleotides: implications for therapeutic application within the central nervous system. Proc Natl Acad Sci USA 90:4665-4669, 1993

32. Zhang ET, Richards HK, Kida S, et al: Directional and compartmentalised drainage of interstitial 
fluid and cerebrospinal fluid from the rat brain. Acta Neuropathol 83:233-239, 1992

33. Zhang R, Lu Z, Zhao H, et al: In vivo stability, disposition and metabolism of a "hybrid" oligonucleotide phosphorothioate in rats. Biochem Pharmacol 50:545-556, 1995

Manuscript received September 15, 1997.

Accepted in final form October 14, 1997.

This work was supported in part by the Huston Foundation, the Lind Lawrence Foundation, the Emily S. and Coleman A. Hunter Trust, and NIH Grant No. K08-NS0176.

Address reprint requests to: William C. Broaddus, M.D., Ph.D., Division of Neurosurgery, Medical College of Virginia, PO Box 980631, Richmond, Virginia 23298-0631. 